\title{
Parâmetros metabólicos de ratos wistar submetidos à dieta suplementada com estévia e açúcar
}

\section{Metabolic parameters of wistar rats subjected to diet supplemented with stevia and sugar}

\author{
Tatiana Abreu Reis ${ }^{1}$; Patrícia de Fátima Pereira Goulart ${ }^{2 *}$; Roseane Maria \\ Evangelista de Oliveira ${ }^{3}$; Lílian de Oliveira ${ }^{4}$; Priscilla Silva de Abreu $^{5}$; Adolfo de \\ Oliveira Azevedo ${ }^{6}$
}

\section{Resumo}

O objetivo da presente pesquisa foi avaliar o ganho de peso, perfil lipídico, glicemia de jejum, função hepática e avaliação histológica de vísceras de ratos wistar submetidos a dietas especiais esperando estabelecer uma análise comparativa entre a estévia e a sacarose, perfazendo os seguintes tratamentos: T1 (controle); T2 (sacarose 4\%); T3 (estévia 2\%); T4 (estévia 4\%) e T5 (estévia 6\%) por 45 dias. Não houve significância para ganho de peso, consumo médio da ração, níveis de triglicérides, colesterol total e VLDLc, uréia e creatinina, independente da dieta ingerida. Os animais dos tratamentos T3 e T4 apresentaram menores níveis de glicose. Os animais do T2 apresentaram valor médio inferior, para o HDLc, enquanto que no LDLc e na ALT, o valor médio encontrado foi superior aos demais. Os animais, dos tratamentos T1 e T5, apresentaram níveis médios de AST inferiores aos demais. As lâminas histológicas apresentaram uma camada de gordura nas vísceras (rins) dos animais do tratamento T2 e nos demais animais dos outros tratamentos essa característica não foi observada. Neste contexto, a estévia apresenta características benéficas para a saúde, principalmente quando comparada a sacarose, podendo substituir o açúcar.

Palavras-chave: Perfil lipídico, função renal e hepática, ganho de peso, estévia

\begin{abstract}
The aim of this study was to evaluate weight gain, lipid profile, fasting glucose, liver function, and histological evaluation of the organs of wistar rats subjected to special diets hoping to establish a comparative analysis of stevia, and sucrose, comprising the following treatments: T1 (control), T2 (sucrose 4\%), T3 (stevia 2\%) and T4 (stevia 4\%) and T5 (stevia 6\%) for 45 days. There was no significant difference for weight gain, the average consumption of diet, levels of triglycerides, total cholesterol and VLDLc, urea and creatinine levels independent of dietary intake. The animals of T3 and T4 had lower glucose levels. The animals of T2 had a lower average value for HDLc, LDLc, while in the AST, the mean value was higher than others. Animals, treatments T1 and T5, showed average levels of ALT inferior to the others. The histological sections showed a layer of fat in the viscera (kidney) of animals of $\mathrm{T} 2$ and for the other animals from other treatments that feature was not observed. In this context, stevia has beneficial characteristics to health and can replace sugar.
\end{abstract}

\footnotetext{
1 Discente Mestranda da Universidade Federal de Lavras, UFLA, Lavras, MG. E-mail: tatianaabreureis@yahoo.com.br

2 Prof $f^{a}$. Pesquisadora Centro Universitário de Lavras, Lavras, MG. E-mail: pat.goulart@hotmail.com

Discente Doutoranda da UFLA, Lavras, MG. E-mail: rmeevangelista@hotmail.com

4 Prof ${ }^{a}$. Pesquisadora do Centro Universitário de Lavras, Lavras, MG. E-mail: lilian@unilavras.edu.br

5 Discente, Mestranda do Centro Universitário de Lavras, Lavras, MG. E-mail: priscillabreu@gmail.com

6 Prof. Pesquisador do Centro Universitário de Lavras, Lavras, MG. E-mail adolfoazevedo@unilavras.edu.br

* Autor para correspondência 
Key words: Lipid profile, renal and hepatic function, weight gain, stevia

\section{Introdução}

O elevado padrão da sociedade tecnológica tem mudado o conceito de saúde, e a introdução de novos produtos químicos tem modificado os hábitos alimentares do homem, que está procurando cada vez mais um modo de vida saudável. Por simples problemas de estética ou por problemas de saúde, o homem está substituindo o conhecido e consagrado açúcar (sacarose) por produtos conhecidos como edulcorantes compostos por sabor semelhante à sacarose, porém com baixo valor calórico ou completamente sem calorias (HEIKAL et al., 2008; KAUSHIK et al., 2010).

A maioria dos edulcorantes tem o inconveniente de apresentar um ligeiro sabor residual desagradavelmente amargo após a ingestão. Nesse sentido, as indústrias estão buscando mais alternativas para possibilitar ao comensal desfrutar o sabor dos alimentos sem precisar sair da dieta (FRONZA, 2002).

No Brasil, os edulcorantes aprovados pela Agencia Nacional de Vigilância Sanitária (ANVISA), para uso em adoçantes dietéticos não calóricos, são: a sacarina, o ciclamato, o aspartame, o acesulfame-k, a sucralose e o esteviosídeo, entre outros (ANVISA, 2008).

No Brasil e em outros países, existe um grande interesse na pesquisa, plantio, industrialização, comercialização de Stevia rebaudiana (Bert.) Bertoni, isto devido aos seus princípios edulcorantes. Estes apresentam a possibilidade de seu uso em larga escala em alimentos, bebidas e medicamentos. Estes princípios edulcorantes contêm os glicosídeos que são de baixo valor calórico, podendo ser utilizados com sucesso como alternativas do açúcar tradicional para pacientes que sofrem de diabetes e outras doenças relacionadas (MELIS; ROCHA; AUGUSTO, 2009).
As folhas de Stevia rebaudiana (Bert.) Bertoni contêm uma mistura de diterpenos labdanos, triterpenos estigmasterol, taninos, óleos voláteis e oito glicosídeos diterpenos adoçantes, que são: esteviosídeo, rebaudiosídeo A, B, C, D, E e dulcosídeos A e B (MORESCHI, 1999). Destes, o rebaudiosídeo A é o mais doce, o mais estável e menos amargo que o esteviosídeo. O rebaudiosídeo E é tão doce quanto o esteviosídeo, e o rebaudiosídeo D quanto o rebaudiosídeo A, enquanto os outros glicosídeos são menos doces que o esteviosídeo (PASQUEL et al., 2000).

O esteviosídeo e, o rebaudiosídeo $\mathrm{A}$, é o segundo maior componente edulcorante da planta, apresentando 250 a 400 vezes o dulçor da sacarose, possuindo também sabor residual levemente amargo (GOTO; CLEMENTE, 1998).

O metabolismo do esteviosídeo em humanos foi esclarecido a partir da identificação de metabólitos na urina e fezes após sua ingestão (TORLONI et al., 2007).

Segundo Torloni et al. 2007, a preferência pelo sabor doce é uma característica inerente a todos os seres humanos. O açúcar (sacarose) consiste na combinação de duas substâncias (glicose e frutose) e fornece $4 \mathrm{kcal} / \mathrm{g}$. O sabor doce é percebido em soluções contendo no mínimo $1 \%$ a $2 \%$ de sacarose. O açúcar é adicionado a diversos alimentos industrializados por seu efeito conservante (antioxidante e antimicrobiano), por proporcionar uma cor agradável aos produtos por meio da caramelização e melhorar a textura dos alimentos processados, modificando sua maciez e viscosidade. No entanto, de acordo com estudos dos cientistas da Associação Americana de Medicina, que acompanharam seis mil pessoas durante sete anos, e revelaram que o consumidor não deve apenas se preocupar com a gordura para "evitar problemas no coração". O excesso de açúcar também pode ser 
responsável pelo aumento dos níveis de colesterol entre outras disfunções (SANTIAGO; KOBAYASI, 2008; BORTSOV et al., 2011; DISHCHEKENIAN et al., 2011).

Portanto, diante do exposto, o presente estudo teve por objetivo, uma vez que é um tema atual e de interesse da comunidade científica, avaliar os resultados obtidos do perfil lipídico, glicemia de jejum, função hepática, função renal e o ganho de peso de ratos wistar submetidos à dieta suplementada com estévia e açúcar, esperando que grandes contribuições possam ser repassadas, no sentido de esclarecer melhor a utilização de um edulcorante natural, como a estévia, sendo um substituto do açúcar e posteriormente contribuir para uma base estruturada, dando continuidade a futuros estudos nesta linha de pesquisa.

\section{Materiais e Métodos}

\section{Delineamento experimental}

O presente estudo foi conduzido utilizandose a estrutura do Laboratório II de Pesquisa com animais, anexo do Laboratório Multidisciplinar de Experimentação Animal e o Laboratório Multidisciplinar de Nutrição, do Centro Universitário de Lavras - UNILAVRAS, seguindo o protocolo experimental do Colégio Brasileiro de Experimentação Animal e aprovado pelo Comitê de Ética em Experimentação Animal do UNILAVRAS.

\section{Animais de experimentação}

Foram utilizados 20 ratos wistar machos $( \pm 100 \mathrm{~g})$, provenientes de matriz pertencente ao Biotério anexo do Laboratório Multidisciplinar de Experimentação Animal do UNILAVRAS, (quatro semanas), separados em grupos de quatro animais perfazendo cinco tratamentos: T1 (controle); T2 (sacarose 4\%); T3 (estévia 2\%); T4 (estévia 4\%) e T5 (estévia 6\%). Os animais foram acondicionados em gaiolas individuais, no Laboratório II de pesquisa com animais, dispostas uma ao lado da outra em prateleiras e mantidos em ambiente controlado com ciclo claro-escuro de doze horas, a $23^{\circ} \mathrm{C}$ e $55 \%$ de umidade. Os animais tiveram acesso a ração e água potável ad libitum.

\section{Estévia, sacarose e dieta experimental}

A estévia, da marca Stevita, fabricada por Steviafarma Industrial S.A. e a sacarose, da marca Monte Alegre, produzida pela Usina Monte Alegre, foram adquiridas no comércio local e ministradas em uma dieta experimental à base de fubá de milho (mimoso) da marca Pachá, fabricado por Arcos Comércio e Importação Ltda., na forma de angu, que foi elaborada com uma composição diferenciada em relação aos teores de carboidrato, especificamente as concentrações de sacarose.

A dieta experimental constituída por angu (fubá e água em proporções equivalentes a um angu feito tradicionalmente na culinária) estévia e sacarose foi produzida no Laboratório Multidisciplinar de Nutrição, onde foram pesados e balanceados todos os ingredientes propostos, de acordo com tipo de tratamento, elaborada para seis dias e mantida sob refrigeração em frascos hermeticamente fechados. Diariamente, os animais recebiam a dieta quantificada, sendo $10 \mathrm{~g}$ de dieta experimental para cada $100 \mathrm{~g}$ de peso do animal.

Para o tratamento um (T1), na dieta experimental utilizou-se angu (controle), sem adição de estévia ou açúcar.

Para o tratamento dois (T2), na dieta experimental utilizou-se angu com adição de açúcar na concentração de $4 \%$.

Já para os tratamentos três (T3), quatro (T4) e cinco (T5), na dieta experimental utilizou-se angu com adição de estévia na concentração de $2 \%, 4 \%$ e $6 \%$, respectivamente.

Após a ministração da dieta experimental (constituída por angu e edulcorantes), que ocorreu 
em todos os quarenta e cinco dias do experimento, os animais também recebiam ração padrão Nuvilab, fabricada por Nuvital Nutrientes S/A, e água ad libitum.

\section{Ganho de peso}

A mensuração do peso dos animais foi realizada diariamente, individualmente, com os animais acomodados em uma caixa de fibra e colocados em uma balança digital, da marca Filizola, tarada, e os valores anotados para controle do peso.

\section{Eutanásia e necrópsia}

As eutanásias foram realizadas no $45^{\circ}$ dia, utilizando a seguinte metodologia: os animais foram anestesiados com hidrato de cloral (dose $1 \mathrm{~g} / \mathrm{kg} ;$ volume $0,5 \mathrm{~mL} / 100 \mathrm{~g}$ animal) pela via intraperitoneal; após a certificação da anestesia ocorreu incisão muscular, divulsão, rompimento da artéria aorta, o sangue foi coletado com uma seringa $5 \mathrm{~mL}$ e depositado no tubo para coleta; e em seguida esse material foi encaminhado para o Laboratório de Análises Clínicas Santa Cecília - Lavras/MG, onde foram determinados glicemia, perfil lipídico, função hepática e renal. Posteriormente, foram necropsiados para a retirada das vísceras para a realização das análises anatomo histopatológicas.

\section{Análises bioquímicas}

\section{Métodos utilizados}

Glicemia: método Oxidase utilizando o Kit Ácido úrico Liquiform ${ }^{\circledR}$; Colesterol total: método enzimático Trinder utilizando o Liquiform ${ }^{\circledR}$; HDLcolesterol (High Density Lipoprotein, Lipoproteína de alta densidade): método Homogêneo direto utilizando o kit HDL LE® (LABTEST DIAGNÓSTICA, 2002).

LDL-colesterol (Low Density Lipoprotein, Lipoproteína de baixa densidade): foram calculados pela fórmula de Friedewald: [LDLcolesterol $]=[$ colesterol total $]-[$ HDL-colesterol $]$ - [triacilglicerois]/5; VLDL-colesterol (Very Low Density Lipoprotein, Lipoproteína de muito baixa densidade): foram calculados pela fórmula: [VLDLcolesterol $]=[$ triacilglicerois $] / 5$ (BACHORIK, RIFKIND; KWITEROVICH, 1999).

Triacilglicerois: método enzimático Trinder utilizando o kit Sera-Pak ${ }^{\circledR}$ Plus (BAYER, 2003).

TGO (Transaminase Glutâmico Oxalacética): método utilizado é o Cinético UV IFCC do kit Liquiform ${ }^{\circledR}$; TGP (Transaminase Glutâmico Pirúvica): método utilizado é o Cinético UV IFCC do kit Liquiform ${ }^{\circledR}$ (LABTEST DIAGNÓSTICA, 2002).

Ácido Úrico: método enzimático Trinder utilizando o kit Sera-Pak® Plus (BAYER, 2003).

Uréia: método utilizado é o enzimático UV do kit Uréia UV; Creatinina: método Cinético utilizando o kit Creatinina K (LABTEST DIAGNÓSTICA, 2002).

\section{Estatística}

Após coleta de todos os dados, os mesmos foram submetidos à análise estatística utilizando o teste Scott - Knott a $5 \%$ de probabilidade, através do programa de análises estatísticas e planejamento de experimentos SISVAR (FERREIRA, 2000).

\section{Resultado e Discussão}

Ganho de peso $x$ consumo médio de ração padrão

As dietas suplementadas com estévia e açúcar associada à ração padrão ad libitum, não interferiram estatisticamente em relação ao ganho de peso (Tabela 1), bem como nos pesos dos órgãos dos animais. Em relação ao valor médio de ração padrão, consumida pelos animais, observou-se que não houve diferenças significativas entre os 
tratamentos estudados $(\mathrm{P}>0,05)$, demonstrando pesquisa. A dieta experimental teve um consumo de inexistência de interferência nos resultados da $100 \%$ diariamente.

Tabela 1- Valores médios de ganho de peso e consumo de ração $(\mathrm{g})$ de ratos wistar, submetidos à dieta suplementada à base de estévia e açúcar.

\begin{tabular}{lcc}
\hline & GANHO DE PESO E CONSUMO DE RAÇÃO $(\mathbf{g})$ \\
TRATAMENTOS & Ganho de Peso & Consumo de Ração \\
\hline CONTROLE & $315,54 \mathrm{a}( \pm 27,26)$ & $31,37 \mathrm{a}( \pm 2,71)$ \\
AÇÚCAR 4\% & $288,86 \mathrm{a}( \pm 24,96)$ & $28,95 \mathrm{a}( \pm 2,50)$ \\
ESTÉVIA 2\% & $307,37 \mathrm{a}( \pm 26,56)$ & $30,70 \mathrm{a}( \pm 2,65)$ \\
ESTÉVIA 4\% & $314,12 \mathrm{a}( \pm 27,14)$ & $31,37 \mathrm{a}( \pm 2,71)$ \\
ESTÉVIA 6\% & $306,35 \mathrm{a}( \pm 26,47)$ & $30,63 \mathrm{a}( \pm 2,65)$ \\
ERRO PADRÃO & 13,24 & 1,32 \\
CV & 8,64 & 8,64 \\
\hline
\end{tabular}

Os valores representam as médias e \pm o desvio padrão da média.

Médias seguidas de mesmas letras minúsculas na coluna não diferem entre si pelo teste Scott - Knott a 5\% de probabilidade.

\section{Observação macroscópica dos rins}

Nas figuras 1 e 2 estão demonstrados os resultados das lâminas histológicas de ratos wistar, onde se observou que, para os animais que consumiram a dieta experimental $\mathrm{T} 2$, os rins apresentaram uma camada de gordura envolvendo essa víscera, o mesmo não foi observado nos rins dos animais que receberam a dieta experimental T4, as demais vísceras não apresentaram alterações. Portanto, sugere-se que o consumo da estévia seja uma opção saudável ao ser comparado ao açúcar, quando se considera a gordura visceral.

Figura 1. Lâminas de cortes histológicos dos rins dos animais no tratamento T2 (açúcar 4\%).
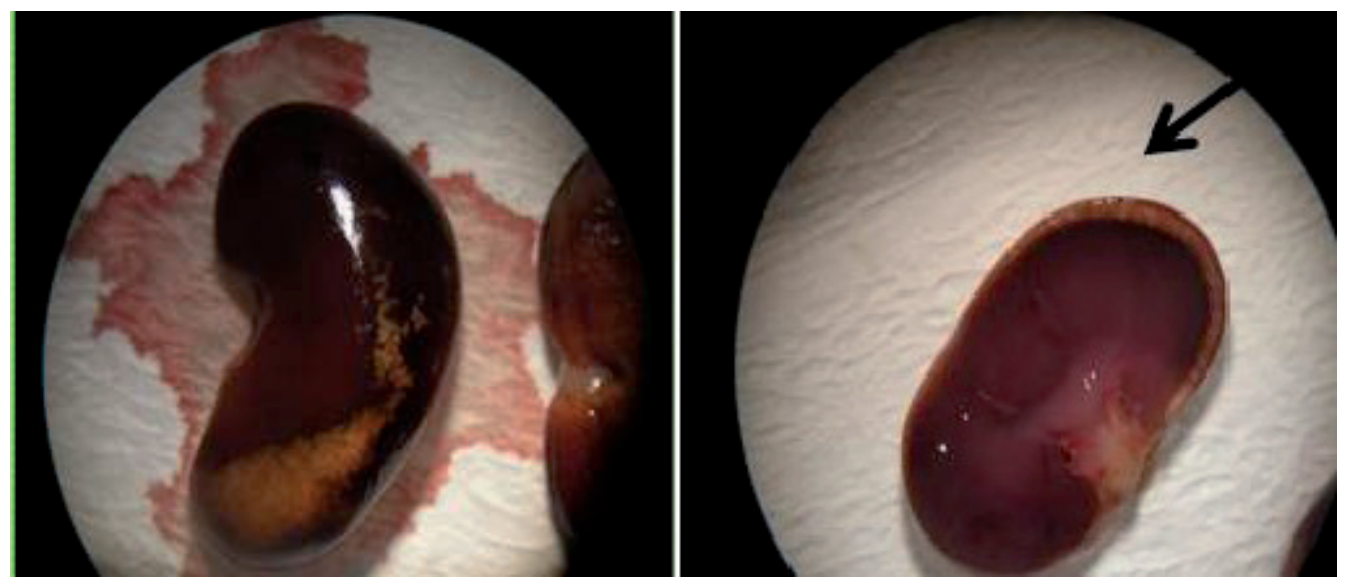
Figura 2. Lâminas de cortes histológicos dos rins dos animais no tratamento T4 (estévia 4\%).
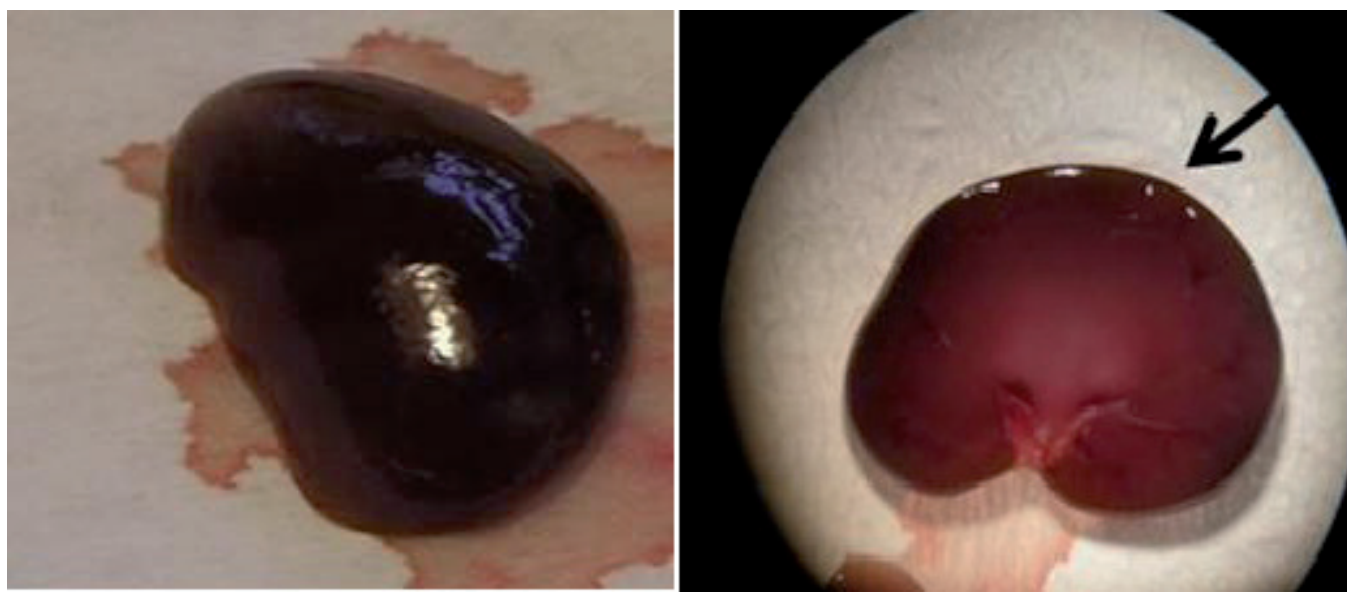

\section{Glicemia de jejum}

Houve diferenças significativas $(\mathrm{P}<0,01)$ nos valores médios dos níveis de glicemia de jejum $(\mathrm{mg} / \mathrm{dL})$ de ratos wistar, submetidos à dieta suplementada com estévia e açúcar. Os animais dos tratamentos T3 e T4 apresentaram menores níveis de glicose em relação aos animais dos tratamentos T2, T5, e T1 respectivamente (Tabela 2). De acordo com Gregersen et al. (2004), em seus estudos relacionados aos efeitos agudos do esteviosídeo em pacientes diabéticos tipo 2, concluíram que a estévia reduz os níveis sanguíneos de glicose pós- prandial em diabéticos tipo 2, indicando efeitos benéficos da estévia sobre o metabolismo da glicose. Ainda em estudos mais recentes, (HOSSIAN et al., 2011; KUJUR et al., 2010) reforçam os efeitos hipoglicemiantes da estévia. Em relação aos resultados encontrados na presente pesquisa, a estévia atuou como agente hipoglicemiante principalmente quando comparada ao tratamento controle e a outros estudos com parâmetros de glicemia (SANTOS et al., 2010; DANTAS et al., 2006).

Tabela 2. Valores médios de glicemia de jejum $(\mathrm{mg} / \mathrm{dL})$ de ratos wistar, submetidos à dieta suplementada com estévia e açúcar.

\begin{tabular}{lc}
\hline & GLICEMIA $(\mathbf{m g} / \mathbf{d L})$ \\
\hline TRATAMENTOS & GLICOSE \\
\hline T1 - Controle & $141,25 \mathrm{c}( \pm 9,08)$ \\
T2 - Açúcar 4\% & $122,25 \mathrm{~b}( \pm 7,86)$ \\
T3 - Estévia 2\% & $110,75 \mathrm{a}( \pm 7,12)$ \\
T4 - Estévia 4\% & $102,75 \mathrm{a}( \pm 6,60)$ \\
T5 - Estévia 6\% & $126,25 \mathrm{~b}( \pm 8,11)$ \\
\hline ERRO PADRÃO & 3,88 \\
\hline CV & 6,43 \\
\hline
\end{tabular}

Os valores representam as médias e \pm o desvio padrão da média.

Médias seguidas de mesmas letras minúsculas na coluna não diferem entre si pelo teste Scott - Knott a 5\% de probabilidade. 


\section{Perfil lipídico}

$\mathrm{Na}$ tabela 3, estão demonstrados os valores médios dos níveis do perfil lipídico de ratos wistar, submetidos à dieta suplementada com estévia e açúcar. Observa-se que não houve diferenças significativas $(\mathrm{P}>0,05)$ entre os tratamentos para os níveis de triglicérides, colesterol total e VLDLc. Já, em relação ao HDLc, os animais do tratamento $\mathrm{T} 2$, apresentaram valor médio significativamente inferior $(\mathrm{P}<0,01)$ aos animais dos demais tratamentos. Observa-se que para o LDLc, o valor médio encontrado foi significativamente superior $(\mathrm{P}<0,01)$, nos animais do tratamento $\mathrm{T} 2$, enquanto que os animais dos tratamentos suplementados com estévia não sofreram alterações, indicando possíveis benefícios em seu consumo ao ser comparado ao açúcar.

Tabela 3. Valores médios do perfil lipídico $(\mathrm{mg} / \mathrm{dL})$ de ratos wistar, submetidos à dieta suplementada com estévia e açúcar.

\begin{tabular}{lccccc}
\hline \multicolumn{7}{c}{ PERFIL LIPÍDICO $(\mathbf{m g} / \mathbf{d L})$} \\
\hline TRATAMENTOS & TG & CT & HDLc & LDLc & VLDLc \\
\hline T1 - Controle & $50,00 \mathrm{a}( \pm 7,71)$ & $58,50 \mathrm{a}( \pm 4,43)$ & $29,00 \mathrm{~b}( \pm 2,14)$ & $19,50 \mathrm{a}( \pm 2,57)$ & $10,00 \mathrm{a}( \pm 1,54)$ \\
T2 - Açúcar 4\% & $48,75 \mathrm{a}( \pm 7,52)$ & $57,50 \mathrm{a}( \pm 4,35)$ & $19,00 \mathrm{a}( \pm 1,40)$ & $28,75 \mathrm{~b}( \pm 3,79)$ & $9,75 \mathrm{a}( \pm 1,50)$ \\
T3 - Estévia 2\% & $46,25 \mathrm{a}( \pm 7,13)$ & $60,00 \mathrm{a}( \pm 4,54)$ & $29,00 \mathrm{~b}( \pm 2,14)$ & $21,75 \mathrm{a}( \pm 2,86)$ & $9,25 \mathrm{a}( \pm 1,42)$ \\
T4 - Estévia 4\% & $51,25 \mathrm{a}( \pm 7,90)$ & $56,75 \mathrm{a}( \pm 4,30)$ & $27,25 \mathrm{~b}( \pm 2,01)$ & $19,25 \mathrm{a}( \pm 2,53)$ & $10,25 \mathrm{a}( \pm 1,18)$ \\
T5 - Estévia 6\% & $57,50 \mathrm{a}( \pm 8,87)$ & $59,00 \mathrm{a}( \pm 4,47)$ & $26,25 \mathrm{~b}( \pm 1,93)$ & $21,25 \mathrm{a}( \pm 2,80)$ & $11,50 \mathrm{a}( \pm 1,77)$ \\
\hline ERRO PADRÃO & 3,91 & 2,21 & 0,96 & 1,45 & 0,78 \\
\hline CV & 15,42 & 7,57 & 7,37 & 13,17 & 15,42 \\
\hline
\end{tabular}

Os valores representam as médias e $\pm \mathrm{o}$ desvio padrão da média.

Médias seguidas de mesmas letras minúsculas na coluna não diferem entre si pelo teste Scott - Knott a 5\% de probabilidade.

Os resultados encontrados na presente pesquisa relacionados aos níveis de colesterol total não diferiram entre os tratamentos. Foi verificado, porém, que os níveis de HDLc reduziram e os níveis de LDLc aumentaram nos animais dos tratamento T2 (4\%), o que justificou o nível de colesterol total não diferir dos animais dos demais tratamentos. No entanto, não é o desejável, pois, níveis reduzidos de HDLc e aumentados de LDLc estão diretamente relacionados a incidência de doenças coronarianas.

Esses resultados corroboram com Appleton (2010), o qual relata que além de destruir a homeostase do organismo, o açúcar pode provocar um aumento significativo no nível total de colesterol, triglicerídeos e LDLc e reduzir o HDLc.
Também de acordo com estudos dos cientistas da Associação Americana de Medicina, que acompanharam seis mil pessoas durante sete anos, e revelaram que o consumidor não deve apenas se preocupar com a gordura para "evitar problemas no coração". O excesso de açúcar também pode ser responsável pelo aumento dos níveis de colesterol (DISHCHEKENIAN et al., 2011).

\section{Função renal}

De acordo com os valores médios da função renal, os parâmetros bioquímicos avaliados na urina dos animais, submetidos à dieta suplementada com estévia e açúcar, não apresentaram diferença 
mínima significativa $(\mathrm{P}>0,05)$, para os níveis de uréia e creatinina independente da dieta ingerida.

Os valores de uréia se assemelham aos observados por Dantas et al. (2006) e Centro de Bioterismo da FUMSP, (2008), mas diferem dos valores encontrados por Santos et al. (2010).

Para a creatinina, os valores encontrados, foram semelhantes aos observados por Dantas et al. (2006), Harkness e Wagner (1993) e Centro de
Bioterismo da FUMSP, (2008), permitindo inferir que os tratamentos não interferiram diretamente na função renal. Segundo Al-habori et al. (2002) apud Teixeira et al., (2009), a creatinina é o principal indicador da função renal, sendo que a elevação dos níveis de creatinina no sangue é observada principalmente quando há lesão dos néfrons funcionantes.

Tabela 4. Valores médios da função renal (U/L) de ratos wistar, submetidos à dieta suplementada com estévia e açúcar.

\begin{tabular}{lcc}
\hline \multicolumn{3}{c}{ FUNÇÃO RENAL $(\mathbf{m g} / \mathbf{d L})$} \\
\hline TRATAMENTOS & URÉIA & CREATININA \\
\hline CONTROLE & $42,50 \mathrm{a}( \pm 5,763)$ & $0,45 \mathrm{a}( \pm 0,064)$ \\
AÇÚCAR 4\% & $47,75 \mathrm{a}( \pm 6,475)$ & $0,38 \mathrm{a}( \pm 0,054)$ \\
ESTÉVIA 2\% & $46,75 \mathrm{a}( \pm 6,339)$ & $0,40 \mathrm{a}( \pm 0,057)$ \\
ESTÉVIA 4\% & $44,50 \mathrm{a}( \pm 6,034)$ & $0,40 \mathrm{a}( \pm 0,057)$ \\
ESTÉVIA 6\% & $41,75 \mathrm{a}( \pm 5,661)$ & $0,35 \mathrm{a}( \pm 0,050)$ \\
\hline ERRO PADRÃO & 3,03 & 0,03 \\
\hline CV & 13,56 & 14,25 \\
\hline
\end{tabular}

Os valores representam as médias e \pm o desvio padrão da média.

Médias seguidas de mesmas letras minúsculas na coluna não diferem entre si pelo teste Scott - Knott a 5\% de probabilidade.

\section{Função hepática}

Nos resultados relacionados aos valores médios da função hepática (U/L) de ratos wistar, submetidos à dieta suplementada com estévia e açúcar (Tabela 5), verificou-se que os animais do tratamento T2, apresentaram níveis de médios de ALT significativamente $(\mathrm{P}<0,05)$ superior aos animais dos demais tratamentos.

Porém, para os níveis médios de AST, os animais dos tratamentos $\mathrm{T} 1$ e $\mathrm{T} 5$, foram os que apresentaram níveis significativamente $(\mathrm{P}<0,05)$ inferiores aos demais tratamentos. Para a enzima ALT, a média geral encontrada (Tabela 5) demonstrou-se acima dos valores de referência, para ratos, uma vez que o tratamento T2 apresentou-se muito elevado em relação aos demais tratamentos. Já a enzima AST (Tabela 5), demonstrou diferenças estatísticas entre os tratamentos, uma vez que os tratamentos $\mathrm{T} 2$, $\mathrm{T} 3$ e T4 apresentaram resultados superiores aos demais tratamentos. 
Tabela 5. Valores médios da função hepática (U/L) de ratos wistar, submetidos à dieta suplementada com estévia e açúcar.

\section{FUNÇÃO HEPÁTICA (U/L)}

\begin{tabular}{llc}
\hline TRATAMENTOS & ALT & AST \\
\hline T1 - CONTROLE & $71,25 \mathrm{a}( \pm 2,86)$ & $273,00 \mathrm{a}( \pm 17,44)$ \\
T2 - AÇÚCAR 4\% & $111,75 \mathrm{~b}( \pm 4,48)$ & $339,00 \mathrm{~b}( \pm 21,66)$ \\
T3 - ESTÉVIA 2\% & $70,25 \mathrm{a}( \pm 2,82)$ & $315,00 \mathrm{~b}( \pm 20,13)$ \\
T4 - ESTÉVIA 4\% & $72,50 \mathrm{a}( \pm 2,91)$ & $343,75 \mathrm{~b}( \pm 21,97)$ \\
T5 - ESTÉVIA 6\% & $68,00 \mathrm{a}( \pm 2,73)$ & $301,50 \mathrm{a}( \pm 19,27)$ \\
\hline ERRO PADRÃO & 1,58 & 10,04 \\
\hline CV & 4,01 & 6,39 \\
\hline
\end{tabular}

Os valores representam as médias e \pm o desvio padrão da média.

Médias seguidas de mesmas letras minúsculas na coluna não diferem entre si pelo teste Scott - Knott a 5\% de probabilidade.

Apesar da diferença significativa, a média geral encontrada apresentou valores bioquímicos aceitáveis, de acordo com os valores de referência proposto por Dantas et al. (2006). Já os valores de ALT foram maiores quando comparados com os encontrados por Teixeira et al. (2009). Os mesmos autores ainda ressaltam que a ALT é um indicador mais sensível de hepatoxicidade do que a AST, pois sabe-se que a AST existe em todos os tecidos corporais, especialmente no coração, fígado, músculo esquelético, rins, cérebro, pâncreas, leucócitos, eritrócitos, já a ALT é encontrada primariamente no fígado, e em pequena quantidade no rim e no coração. Estas enzimas são liberadas no sangue em grandes quantidades quando há dano à membrana do hepatócito, resultando em aumento da permeabilidade (LAB REDE, 2008). Porém, até o momento não há estudos que relacione o aumento dessas enzimas com a ingestão de açúcar bem como a estévia.

\section{Conclusão}

Não houve diferenças significativas $(\mathrm{P}>0,05)$ para o ganho de peso, consumo médio da ração padrão, níveis de triglicérides, colesterol total e
VLDLc, uréia e creatinina, independente da dieta ingerida.

Os animais dos tratamentos $\mathrm{T} 3 \mathrm{e} \mathrm{T} 4$ apresentaram menores níveis de glicose em relação aos animais dos tratamentos T2, T5, e o T1, respectivamente.

Os animais do T2 apresentaram valor médio inferior $(\mathrm{P}<0,01)$, para o HDLc, enquanto que no LDLc e na ALT, o valor médio encontrado foi superior $(\mathrm{P}<0,01)$ aos demais.

Os animais dos tratamentos T1 e T5 apresentaram níveis médios de AST $(\mathrm{P}<0,05)$ inferiores aos demais.

As lâminas histológicas das vísceras (rins) apresentaram uma camada de gordura nos animais do tratamento T2, quando comparados aos animais dos demais tratamentos, as demais vísceras não apresentaram alterações.

\section{Agradecimento}

A FAPEMIG, CNPq e ao UNILAVRAS, pelo apoio à pesquisa e concessão de bolsas. 


\section{Referências}

AGÊNCIANACIONAL DE VIGILÂNCIA SANITÁRIA - ANVISA. Apresenta informações sobre saúde nacional. Disponível em: <http://www.anvisa.gov.br > . Acesso em: 18 de maio 2008.

AL-HABORI, M.; AL-AGHBARI, A.; AL-MAMARY, M.; BAKER, M. Toxicological evaluation of Catha edulis leaves: a long term feeding experiment in animals. $J$ Ethnopharmacol, Taipei,Taiwan, v. 83, n. 3, p. 209217, 2002.

APPLETON, N. 76 Modos do açúcar acabar com a saúde. Disponível em: <http://www.guiavegano.com/ nutricao/nancy/76modos.htm>. Acesso: 29 abr. 2010.

BACHORIK, P. S.; RIFKIND, B. M.; KWITEROVICH, P. O. Lipídios e dislipoproteinemias. In: HENRY, J. B. Diagnóstico clínico e tratamento por métodos laboratoriais. 9. ed. São Paulo: Manole, 1999. p. 208236.

BAYER. Sera-Pak®. Plus colesterol e plus triacilglicerois. Argentina, Buenos Aires: UFSC, 2003.

BORTSOV, A. V.; LIESE, A. D.; BELL, R. A., DABELEA, D.; D'AGOSTINO JUNIOR, R. B.; HAMMAN, R. F.; KLINGENSMITH, G. J.; LAWRENCE, J. M.; MAAHS, D. M.; MCKEOWN, R.; MARCOVINA, S. M.; THOMAS, J.; WILLIAMS, D. E.; MAYER-DAVIS, E. J. Sugar-sweetened and diet beverage consumption is associated with cardiovascular risk factor profile in youth with type 1 diabetes. Acta Diabetol, Milão, Itália, jan. 2011. Disponível em: $<$ http:// www.springerlink.com/content/600075863470145h/>. Acesso em 13 maio 2010.

CENTRO DE BIOTERISMO DA FMUSP. Parâmetros bioquímicos de ratos do centro de bioterismo da FMUSP. 2008. Disponível em: $<\mathrm{http}: / /$ www.biot.fm.usp. br/index.php?mpg=03.00.00\&tip=RATO\&id_ani= 17\&parametros $=\operatorname{sim}>$. Acesso em: 12 maio 2010.

DANTAS, A. J.; AMBIEL, C. R.; CUMAN, R. K. N.; BARONI, S.; BERSANI-AMADO, C. A. Valores de referência de alguns parâmetros fisiológicos de ratos do biotério Central da Universidade Estadual de Maringá, Estado do Paraná. Acta Science Health Science, Maringá, v. 28 , n. 2, p. 165-170, 2006.

DISHCHEKENIAN, V. R. M.; ESCRIVÃO, M. A. M. S.; PALMA, D.; ANCONA-LOPEZ, F.; ARAÚJO, E. A. C.; TADDEI, J. A. A. C. Padrões alimentares de adolescentes obesos e diferentes repercussões metabólicas. Rev. Nutr., Campinas, v. 24, n. 1, p. 17-29, jan./fev. 2011.

FERREIRA, D. F. Análises estatísticas por meio do SISVAR para Windows versão 4.0. In: REUNIÃO
ANUAL DA REGIÃO BRASILEIRA DA SOCIEDADE INTERNACIONAL DE BIOMETRIA, 45., São Carlos. Anais... São Carlos, SP: UFSCar, p. 255-258, 2000.

FRONZA, D. Consumo de água da cultura de estévia (Stevia rebaudiana (Bert.) Bertoni) estimado por microlisímetro automático. 2002. Tese (Doutorado em Irrigação e Drenagem) - Escola Superior de Agricultura Luiz de Queiroz, Piracicaba.

GOTO, A.; CLEMENTE, E. Influência do rebaudiosídeo A na solubilidade e no sabor do esteviosídeo. Ciência e Tecnologia de Alimentos, Campinas, v. 18, n. 1, p. 3-6, jan./apr. 1998.

GREGERSEN, S.; JEPPESEN, P. B.; HOLST, J. J.; HERMANSEN, K. Antihyperglycemic effects of stevioside in type 2 diabetic subjects. Metabolis, Indonésia, v. 53, n. 1, p. 73-6, 2004.

HARKNESS, J. E.; WAGNER, J. E. Biologia e clínica de coelhos e roedores. 3. ed. São Paulo: Editora Roca, 1993.

HEIKAL, A. H.; BADAWY, O. M.; HAFEZ, A. M. Genetic relationships among some Stevia (Stevia Rebaudiana Bertoni) accessions based on ISSR analysis. Research Journal of Cell and Molecular Biology, Alexandria, Egito, v. 2, n. 1, p. 1-5, 2008.

HOSSIAN, M. S.; ALAM, M. B.; ASADUJJAMAN, M.; MONIRUL ISLAM, M.; RAHMAN, A. M.; ISLAM, A.; ISLAM, A. Antihyperglycemic and anttihyperlipidemic effects of different fractions of stevia rebaudiana leaves in alloxan induced diabetic rats. International Journal of Pharmaceutical Sciences and Research, Jhansi,India, v. 2, n. 7, p. 1722-1729, 2011.

KAUSHIK, R.; PRADEEP, N.; VASUDEVAN, V.; GEETHA, M.; USHA, A. Nutrient composition of cultivated stevia leaves an the influence of polyphenols and plant on sensory and antioxidant properties of leaf extracts. Journal of Food Science Tecnology, Bangalore, Índia, v. 47, n. 1, p. 27-33, jan./feb. 2010.

KUJUR, R.; SINGH, V.; RAM, M.; YADAVA, H. N.; SINGH, K. K.; KUMARI, S.; ROY, B. K. Antidiabetic activity and phytochemical screening of crude extract of Stevia rebaudiana in alloxan-induced diabetic rats. Pharmacognosy Research, Cork, Irlanda, v. 2, n. 2, p. 58-63, 2010.

LAB REDE. Laboratório de referência em diagnósticos especializados. Disponível em: <http://www.labrede. com.br>. Acesso em: 21 jun. 2009.

LABTEST DIAGNÓSTICA. HDL LE®. Lagoa Santa: Editora Atheneu, 2002.

MELIS, M. S.; ROCHA, S. T.; AUGUSTO, A. Steviol effect, a glycoside of Stevia rebaudiana, on glucose 
clearances in rats. Brazilian Journal of Biology, São Carlos, v. 69, n. 2, p. 371 374, 2009.

MORESCHI, S. R. M. Purificação extrato de Stevia rebaudiana Bertoni através de membranas microporosas. Maringá: PEQ/UEM, 1999. 127 p.

PASQUEL, A.; MEIRELES, M. A. A.; MARQUES, M. O. M.; PETENATE, A. J. Extraction of Stevia glycosides with $\mathrm{CO}_{2}+$ water, $\mathrm{CO}_{2}+$ ethanol, and $\mathrm{CO}_{2}+$ water + ethano. Brazilian Journal of Chemical Engineering, São Paulo, v. 17, n. 3, p. 271-282, 2000.

SANTIAGO, J. R. F.; KOBAYASI, S. Efeito da dilatação gástrica em ratos submetidos à ingestão de água gaseificada sobre a função metabólica hepática. Acta Cirúrgica Brasileira, São Paulo, v. 23 n. 5 p. 412-416, 2008

SANTOS, M. R. V.; SOUZA, V. H.; MENEZES, I. A. C.; BITENCURT, J. L.; REZENDE-NETO, J. M.; BARRETO, A. S.; CUNHA, F. A.; MARÇAL, R. M.; TEIXEIRA-SILVA, F.; QUÍNTANS-JÚNIOR, L. J.; BARBOSA, A. P. O. Parâmetros bioquímicos, fisiológicos e morfológicos de ratos (Rattus novergicus linhagem Wistar) produzidos pelo Biotério Central da Universidade Federal de Sergipe. Scientia Plena, Sergipe, v. 6, n. 10, p. 1-6, 2010.

TEIXEIRA, L. T. A.; JÚNIOR, L. S. S.; QUEIROZ, F. M.; OLIVEIRA, C. N.; SCHWARZ, A. Avaliação de efeitos toxicológicos e comportamentais da Hypericum perforatume da Piper methysticum em ratos. Revista Brasileira de Toxicologia, São Paulo, v. 22, n. 1/2, p. 4249, 2009.

TORLONI, M. R.; NAKAMURA, U.; MEGALE, A.; SANCHEZ, V. H. S.; MANO, C.; FUSARO, A. S.; MATTAR, R. O uso de adoçantes na gravidez: uma análise dos produtos disponíveis no Brasil. Revista Brasileira de Ginecologia e Obstetrícia, Rio de Janeiro, v. 29, n. 5, p. 267-275, maio, 2007. 
Reis, T. A. et. al. 